\title{
Elliptical Membranes with Smallest Second Eigenvalue*
}

\author{
By B. Andreas Troesch
}

\begin{abstract}
The elliptic membranes with fixed boundary are determined, for which the second eigenfrequency is a minimum if the area or if the circumference is prescribed. The results are compared with those of some other shapes. A remark is made about the overtones of elliptic membranes.
\end{abstract}

1. Introduction and Problem Statement. Over the years, the classical membrane problem has not lost its fascination. In spite of a large number of investigations (cf. [3] and the references given therein), there still remain many questions that are natural and can be stated in simple terms. We will present here results of limited scope related to two of these questions, namely results on the second eigenvalue of elliptic membranes, which are fixed along the boundary. It is well known that, among the fixed membranes of a given area, the circle has the lowest principal frequency. The circular shape also furnishes the solution to the isoperimetric membrane problem if the length of the perimeter is prescribed instead of the area.

However, for the second eigenvalue, the situation changes. It has been proved by $\mathrm{G}$. Szegö and reported in [4,p. 336] that there does not exist an admissible membrane (i.e., a connected open domain) for which the second eigenvalue attains a minimum, but that the infimum is obtained for the double circle (cf. Fig. 2b). If the membrane is assumed to be convex, then the solution is not known. But the question can then be asked, whether part of the boundary is straight, and there exists some (numerical) evidence that this might indeed be the case. More is known about the second eigenvalue of a membrane with given circumference. As has been proved in [2], the isoperimetric shape exists, and the membrane is bounded by an analytic convex curve. Again, the exact curve is not known, but it is likely to be not far from the oval shape sketched in Fig. 3d.

Considering then the state of the problem, it appears worthwhile to give at least the solution of the isoperimetric problems for the second eigenvalue for a particular shape, namely for ellipses. After a short summary of the very simple situation for rectangles (Section 2), the solution for the ellipse and the comparison with other shapes is presented in Section 3. The notation and the results of [6] will be used throughout. In the last section, we take up a question that is only loosely connected with the main topic. How could the sound of a drum be improved, if it were not round but shaped like an ellipse instead?

2. Extremal Rectangular Membranes. In order to get a feeling for the problem, let us summarize the results for the rectangular membrane with sides $2 a$ and $2 b$

Received September 6, 1972.

AMS (MOS) subject classifications (1970). Primary 35J05, 73K15; Secondary 70J10, 35J25.

Key words and phrases. Linear membrane vibrations, isoperimetric shape, acoustical overtones.

* This work was supported in part by NSF grant GP-22587. 
$(a \geqq b)$. The solution of the membrane equation [6, Eqs. (2.1), (2.2)] for the second mode is simply

$$
\varphi=\sin (\pi x / a) \sin (\pi y / 2 b)
$$

the eigenvalue

$$
\lambda_{2}=\pi^{2}\left(a^{2}+4 b^{2}\right) / 4 a^{2} b^{2},
$$

and the dimensionless quantities of interest here are therefore

$$
\begin{gathered}
\lambda_{2} A=\pi^{2}\left(a^{2}+4 b^{2}\right) / a b, \\
\lambda_{2} L^{2}=\pi^{2}(a+b)^{2}\left(a^{2}+4 b^{2}\right) / a^{2} b^{2} .
\end{gathered}
$$

Their minima are listed in Tables 3 and 4, and the corresponding shapes are shown in Fig. 2a and Fig. 3a.

We observe that the solution for the rectangle points in the right direction: for fixed area $A$, the double square resembles the double circle, and, among convex membranes, the minimum frequency for the rectangle is even surprisingly good, namely, only $2 \%$ above the best (known) value. For fixed perimeter $L$, the minimum does not come near the best value, but at least the indication is clear, that the shape should be only slightly elongated.

3. The Extremal Ellipses. The membrane problem for ellipses was solved a long time ago. An approximate solution can be obtained directly from Fig. 1, which is an enlargement of Fig. 1 in [6]. The dimensionless quantity $\lambda A$ is [6, Eq. (3.8)]

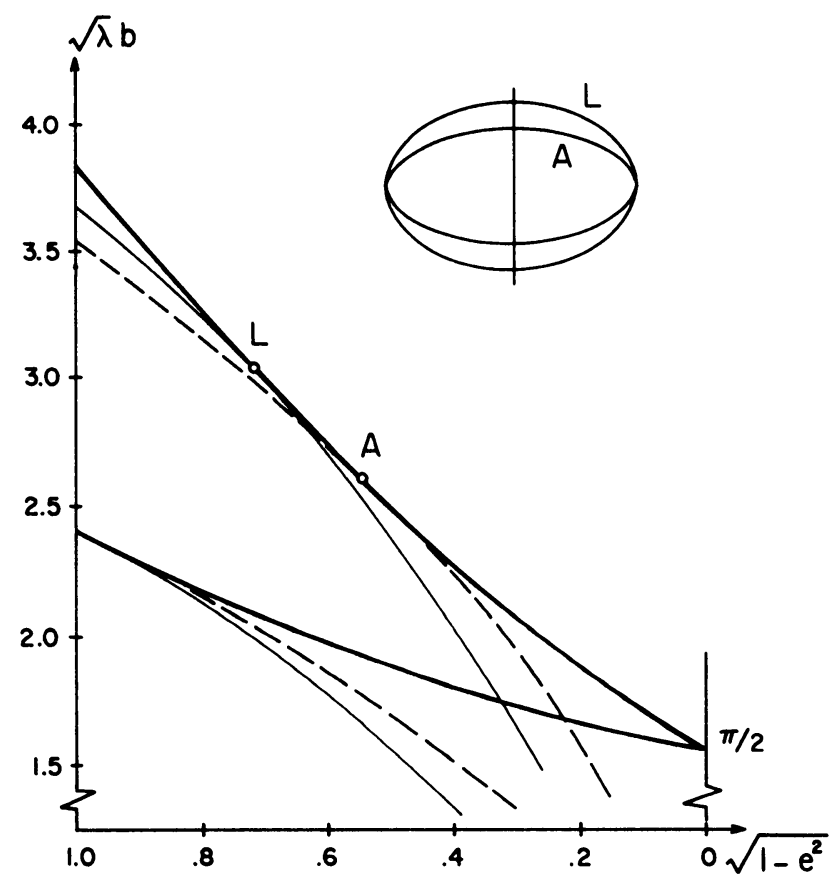

FIGURE 1. The two lowest frequencies of a membrane.___Ellipses of constant area.

- - - Ellipses of constant circumference. A: Point of minimum $\lambda_{2} A$, L: Point of minimum $\lambda_{2} L^{2}$. 


$$
\lambda A=\pi\left(\lambda^{1 / 2} b\right)^{2} /\left(1-e^{2}\right)^{1 / 2} .
$$

Furthermore, the circumference of an ellipse with the major axis $a$ is

$$
L=4 a E,
$$

with $E$ the complete elliptic integral

$$
E=\int_{0}^{\pi / 2}\left(1-e^{2} \sin ^{2} \vartheta\right)^{1 / 2} d \vartheta
$$

It turns out to be a little more convenient to use the quarter-circumference $l=L / 4$, so that

$$
\lambda l^{2}=\left(\lambda^{1 / 2} b\right)^{2} E^{2} /\left(1-e^{2}\right)=\lambda a^{2} E^{2} .
$$

Curves with constant area, Eq. (3.1), and with constant circumference, Eq. (3.2), are plotted in Fig. 1. It is obvious that, in contrast to the principal frequency, the circle is not isoperimetric and the two solutions are different. The shape for a given perimeter is rounder, as was already suggested by the rectangular membrane.

3.1. Ellipses with Given Area. From Fig. 1, the approximate value of $\lambda_{2} A$ is seen to occur at

$$
\lambda^{1 / 2} b \doteq 2.6, \quad\left(1-e^{2}\right)^{1 / 2} \doteq .55
$$

so that (cf. Eq. (3.1))

$$
\lambda_{2} A \doteq 12.3 \pi .
$$

The points 'on the curve of $\lambda_{2}^{1 / 2} b$ are obtained by the method described in [6]. For the extremal membrane, we find that $s \doteq 16$. The results for appropriate $s$ values near it are listed in Table 1, together with

$$
\lambda_{2} A=\pi\left(\lambda^{1 / 2} b\right)\left(\lambda^{1 / 2} a\right) .
$$

The last line gives the interpolated minimum.

In Table 3, we list the data for the best ellipse and for some other shapes. It should be mentioned that the value for the slightly shortened double-square capped by two half-circles of radius $R$ was obtained by the Rayleigh-Ritz method and therefore represents an upper bound.

TABLE 1. The $\lambda_{2} A$ values around the minimum

\begin{tabular}{llccc}
\hline \multicolumn{1}{c}{$s$} & \multicolumn{1}{c}{$\lambda_{2}^{1 / 2} a$} & $\lambda_{2}^{1 / 2} b$ & $\lambda_{2} A / \pi$ & $\lambda_{2} A$ \\
15.5 & 4.7406163 & 2.6407277 & 12.518677 & 39.32858 \\
16.0 & 4.7805548 & 2.6179581 & 12.515292 & 39.31795 \\
16.5 & 4.8207518 & 2.5960831 & 12.515072 & 39.31726 \\
17.0 & 4.8611705 & 2.5750687 & 12.517848 & 38.32598 \\
17.5 & 4.9017769 & 2.5548809 & 12.523456 & 39.34360 \\
18.0 & 4.9425386 & 2.5354857 & 12.531736 & 39.36961 \\
16.2847 & 4.80342 & 2.60539 & 12.51479 & 39.3164 \\
\hline
\end{tabular}


TABLE 2. Numerical results around the minimum of $\lambda_{2} L^{2}$

\begin{tabular}{cccc}
$s$ & \multicolumn{1}{c}{$\lambda_{2}^{1 / 2} a$} & $\lambda_{2}^{1 / 2} b$ & $e^{2}$ \\
7.5 & 4.1714828 & 3.1466282 & .43100 \\
8.0 & 4.2015132 & 3.1068816 & .45319 \\
8.5 & 4.2324488 & 3.0681627 & .47450 \\
9.0 & 4.2642646 & 3.0305037 & .49494 \\
9.5 & 4.2969312 & 2.9939301 & .51452 \\
10.0 & 4.3304148 & 2.9584612 & .53326 \\
8.7693 & 4.24948 & 3.04775 & .48561 \\
\hline
\end{tabular}

3.2. Ellipses with Given Perimeter. The case of the ellipse with a given perimeter and the minimum $\lambda_{2} L^{2}$ is only slightly more complicated. First, we find from Fig. 1 as approximate values

$$
\lambda^{1 / 2} b \doteq 3.0, \quad\left(1-e^{2}\right)^{1 / 2} \doteq .7
$$

and hence

$$
s \doteq 9
$$

The solutions near this value are listed in Table 2. The quarter-circumference $E$ for ellipses with $.42 \leqq e^{2} \leqq .54$ is found in [1, p. 609], and the minimum of $\lambda a^{2} E^{2}$ (see Eq. (3.2)) is interpolated and shown on the last line of Table 2.

\begin{tabular}{|c|c|c|c|}
\hline Fig. 2 & $\lambda_{2} A=f^{2} \pi$ & $f$ & for $\lambda_{2}=1$ \\
\hline (a) & $\begin{aligned} 4 \pi^{2} & =12.5664 \pi \\
& =39.4784\end{aligned}$ & $2 \pi^{1 / 2}=3.54491$ & $\begin{aligned} a & =\pi 2^{1 / 2}=4.4429 \\
b & =a / 2=2.2214 \\
b / a & =.5\end{aligned}$ \\
\hline (b) & $\begin{aligned} 2 j_{0}^{2} \pi & =11.5664 \pi \\
& =36.3368\end{aligned}$ & $j_{0} 2^{1 / 2}=3.40094$ & $\begin{aligned} R & =3.40094 \\
J_{0}\left(j_{0}\right) & =0 \\
j_{0} & =2.4048\end{aligned}$ \\
\hline (c) & $\begin{array}{l}=12.5148 \pi \\
=39.3164\end{array}$ & $=3.53762$ & $\begin{aligned} a & =4.8034 \\
b & =2.6054 \\
b / a & =.54241 \\
e & =.84012\end{aligned}$ \\
\hline (d) & $\begin{array}{l}=12.1292 \pi \\
=38.1050\end{array}$ & $=3.4827$ & $\begin{array}{r}a=4.5687 \\
R=b=2.3429 \\
a-R=2.2258\end{array}$ \\
\hline
\end{tabular}

TABLE 3. Minimum $\lambda_{2} A$ and related quantities 
TABLE 4. Minimum $\lambda_{2} l^{2}$ and related quantities

\begin{tabular}{|c|c|c|c|}
\hline Fig. 3 & $\lambda_{2} l^{2}=\lambda_{2}(L / 4)^{2}$ & \multicolumn{2}{|l|}{ for $\lambda_{2}=1$} \\
\hline (a) & $\begin{array}{l}=4.3304 \pi^{2} \\
=42.7397\end{array}$ & $\begin{aligned} a & =4.0109 \\
b / a & =2^{1 / 3} / 2=.62996\end{aligned}$ & $b=2.5267$ \\
\hline (b) & $\begin{array}{l}=\left(j_{1} / 2\right)^{2} \pi^{2} \\
=36.2264\end{array}$ & $\begin{aligned} R & =j_{1}=3.83171 \\
J_{1}\left(j_{1}\right) & =0\end{aligned}$ & \\
\hline (c) & $\begin{array}{l}=(5.77015)^{2} \\
=33.2946\end{array}$ & $\begin{aligned} a & =4.2495 \\
b / a & =.71721 \\
E & =1.3578\end{aligned}$ & $\begin{array}{l}b=3.0478 \\
e=.69686\end{array}$ \\
\hline (d) & $\begin{array}{l}=(5.7495)^{2} \\
=33.0568\end{array}$ & $a=4.192$ & $b=2.893$ \\
\hline
\end{tabular}

From the results in Table 4, we see that the ellipse does not solve the isoperimetric problem: the value for a certain oval is actually lower. But this conclusion can also be reached from [2, Eq. (4.18)], which establishes the relation between the curvature of the isoperimetric shape and the normal derivative of the second eigenfunction $\varphi$ at the boundary. It is easily shown that if the ellipse were to solve the isoperimetric problem, then the Mathieu function $c e_{1}(q, \eta)$ could be represented as a finite expression of trigonometric functions, and this is clearly not the case.

The method that was used to obtain the results for the oval (essentially an inverse method) is not related to the present investigation and will not be described. But

(a)

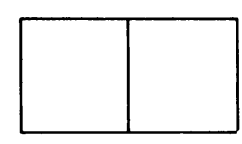

(b)

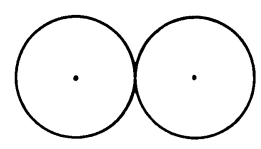

(c)

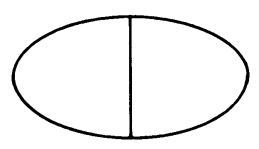

(d)

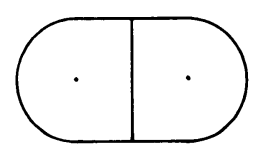

FIGURE 2. The shapes of the membranes listed in Table 3. (a)

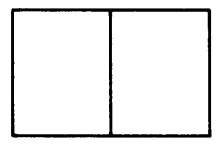

(b)

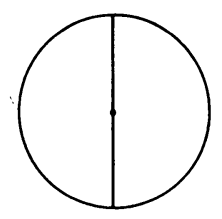

(c)

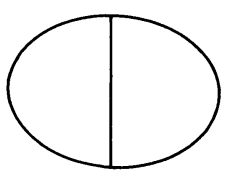

(d)

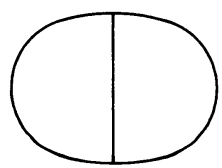

FIGURE 3. The shapes of the membranes listed in Table 4. 
let us mention that the result in Table 4(d) represents (as does 3(d)) an upper bound to the true minimum.

4. A Note on a Harmonious Drum or Timpani. Since the sound of a round drum is not very harmonious, we will show how to construct a better drum or timpani by shaping it as an ellipse. The reason why a string or an organ pipe sounds more pleasant than a drum is obvious; the roots of the trigonometric functions lead to higher harmonics which are simple multiples of the principal frequency, whereas the roots of the Bessel functions do not exhibit this property [5, p. 331]. From the knowledge of the eigenvalues of an elliptical membrane [6, Fig. 1], we can determine the relation of the first few higher harmonics to the principal frequency, and the simpler the ratio the better the sound of the instrument. Now, the simplest ratios are easily seen to be obtained for $e \rightarrow 1$; but this is a degenerate case. However, a real elliptic drum or timpani with $b / a=3.8$ should have a rather pleasant sound. The principal tone and the first three overtones stand very nearly (well within the scale of equal temperament [5, p. 12]), in the ratios of 4:5:6:7 (cf. Fig. 1 for the first two values). This corresponds to the harmony $G B D F$. Furthermore, if the timpani is struck at a point on the major axis, then the two overtones $F^{*}$ and $A$ are not excited, and the following two overtones $\left(G^{\prime \prime}\right.$ and $\left.C^{\prime \prime}\right)$ are hopefully sufficiently weak.

Department of Mathematics

University of Southern California

Los Angeles, California 90007

1. M. Abramowitz \& I. A. Stegun (Editors), Handbook of Mathematical Functions, With Formulas, Graphs and Mathematical Tables, Nat. Bur. Standards Appl. Math. Ser., 55, Superintendent of Documents, U.S. Government Printing Office, Washington, D.C., 1964. MR 29 \#4914.

2. P. R. Garabedian \& M. Schiffer, "Variational problems in the theory of elliptic partial differential equations," J. Rational Mech. Anal., v. 2, 1953, pp. 137-171. MR 14, 984.

3. L. E. PAYNE, "Isoperimetric inequalities and their applications," SIAM Rev., v. 9, 1967 , pp. 453-488. MR 36 \#2058.

4. G. Pólya, "On the characteristic frequencies of a symmetric membrane," Math. Z., v. 63,1955 , pp. 331-337. MR 17, 372 .

5. J. W. S. RAYLEIGH, The Theory of Sound. Vol. 1, 2nd ed., Dover, New York, 1945. MR 7, 500.

6. B. A. Troesch \& H. R. TROESCH, "Eigenfrequencies of an elliptic membrane," Math Comp., v. 27, 1973, pp. 755-765. 